\title{
The Solution Structure of a Chimeric LEKTI Domain Reveals a Chameleon Sequence ${ }^{\dagger}$
}

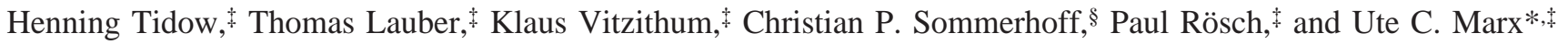 \\ Lehrstuhl für Biopolymere, Universität Bayreuth, Universitätsstrasse 30, 95447 Bayreuth, Germany, and Abteilung für Klinische \\ Chemie und Klinische Biochemie, Klinikumsstandort Innenstadt, Ludwig-Maximilians-Universität, Nussbaumstrasse 20, \\ 80336 München, Germany
}

Received April 16, 2004; Revised Manuscript Received June 25, 2004

\begin{abstract}
The conversion of an $\alpha$-helical to a $\beta$-strand conformation and the presence of chameleon sequences are fascinating from the perspective that such structural features are implicated in the induction of amyloid-related fatal diseases. In this study, we have determined the solution structure of a chimeric domain (Dom1PI) from the multidomain Kazal-type serine proteinase inhibitor LEKTI using multidimensional NMR spectroscopy. This chimeric protein was constructed to investigate the reasons for differences in the folds of the homologous LEKTI domains 1 and 6 [Lauber, T., et al. (2003) J. Mol. Biol. 328, 205-219]. In Dom1PI, two adjacent phenylalanine residues (F28 and F29) of domain 1 were substituted with proline and isoleucine, respectively, as found in the corresponding P4' and P5' positions of domain 6. The three-dimensional structure of Dom1PI is significantly different from the structure of domain 1 and closely resembles the structure of domain 6 , despite the sequence being identical to that of domain 1 except for the two substituted phenylalanine residues and being only $31 \%$ identical to the sequence of domain 6. The mutation converted a short $3_{10}$-helix into an extended loop conformation and parts of the long $\mathrm{COOH}$-terminal $\alpha$-helix of domain 1 into a $\beta$-hairpin structure. The latter conformational change occurs in a sequence stretch distinct from the region containing the substituted residues. Therefore, this switch from an $\alpha$-helical structure to a $\beta$-hairpin structure indicates a chameleon sequence of seven residues. We conclude that the secondary structure of Dom1PI is determined not only by the local protein sequence but also by nonlocal interactions.
\end{abstract}

In general, the information for the three-dimensional structure of most proteins is encoded in the amino acid sequence $(1,2)$. However, there are examples of so-called chameleon sequences which adopt context-dependent secondary structures (3). Identical segments of up to 11 residues can adopt an $\alpha$-helical conformation in one context and a $\beta$-sheet conformation in another (4), whereby nonlocal interactions determine the fold. These conformational changes can be induced by either mutations in a distant sequence position (5), different ligands binding to the same protein (6), or changes in $\mathrm{pH}$ (7). One of the most widely known examples of conversion from an $\alpha$-helix to a $\beta$-sheet conformation occurs in prion diseases, such as transmissible bovine spongiform encephalopathy $(\mathrm{BSE})^{1}(8)$. In inherited human prion diseases, specific mutations are believed to be responsible for the $\alpha \rightarrow \beta$ transition leading to aggregation and amyloid fibrils (9). In this study, we report the alteration of an $\alpha$-helix to a $\beta$-hairpin conformation identified in the solution structure of the chimeric LEKTI domain Dom1PI.

As deduced from the cloned cDNA, the entire LEKTI consists of 1064 amino acid residues containing 15 potential serine proteinase inhibitor domains (10). Due to its predomi-

\footnotetext{
$\dagger$ The study was funded by the Deutshe Forschungsgemeinschaft (DFG) (Project MA2317-1).

* To whom correspondence should be addressed. Phone: ++49 921 552048. Fax: ++49 921 553544. E-mail: ute.marx@uni-bayreuth.de.

$\doteqdot$ Universität Bayreuth.

${ }^{\S}$ Ludwig-Maximilians-Universität.
}

nant expression in lympho-epithelial tissues and its sequential similarity to known Kazal-type inhibitors, the protein was termed lympho-epithelial Kazal-type-related inhibitor (LEKTI). An unequivocal correlation between the severe congenital skin disorder Netherton syndrome and defects in the gene encoding LEKTI (termed SPINK5) was found (1115). Even though the pathophysiological role of LEKTI in Netherton syndrome is undisputed, the actual physiological function of any LEKTI domain remains to be determined. Komatsu et al. (14) localized mRNA encoding LEKTI in the uppermost epidermis of normal human skin. Furthermore, they determined elevated stratum corneum hydrolytic activity in patients with Netherton syndrome, suggesting an inhibitory regulation of desquamation by LEKTI-derived peptides. This is the first hint of a natural target proteinase for any LEKTI domain (14). A role for LEKTI in the regulation of

\footnotetext{
${ }^{1}$ Abbreviations: BMRB, BioMagResBank; BSE, bovine spongiform encephalopathy; DIPSI, decoupling in the presence of scalar interactions; Dom1PI, LEKTI domain 1 double mutant (F28P/F29I); DQFCOSY, double-quantum-filtered correlation spectroscopy; DSS, 2,2dimethyl-2-silapentane-5-sulfonic acid; HNHA, three-dimensional heteronuclear $\left({ }^{1} \mathrm{H}-{ }^{15} \mathrm{~N}-{ }^{1} \mathrm{H}\right)$ shift correlation double-resonance; HSQC, heteronuclear single-quantum coherence; LEKTI, lympho-epithelial Kazal-type-related inhibitor; NMR, nuclear magnetic resonance; NOE, nuclear Overhauser effect (also used for NOESY cross-peak); NOESY, NOE spectroscopy; PDB, Protein Data Bank; RP-HPLC, reversed-phase high-performance liquid chromatography; SPINK5, serine proteinase inhibitor; Kazal-type 5, gene encoding LEKTI; TOCSY, total correlation spectroscopy; Trx, E. coli thioredoxin A; WATERGATE, water suppression by gradient-tailored excitation.
} 
T-lymphocyte differentiation or antimicrobial protection has also been suggested (16).

LEKTI contains two Kazal-type serine proteinase inhibitor domains, as deduced from their primary structure and characteristic pattern of six cysteine residues, together with 13 other domains containing only four of the six cysteines typical of Kazal-type inhibitors. As single domains 1, 5, and 6 have been isolated from ultrafiltrates of human blood (10, 17) and a $30 \mathrm{kDa}$ LEKTI fragment corresponding to approximately four domains starting with domain 8 was isolated from human epidermal keratinocyte-conditioned media (18), we suggest that both single- and multiple-domain fragments of LEKTI occur naturally.

Testing of various serine proteinases showed that domain 6 is an efficient $\left(\mathrm{IC}_{50} \cong 150 \mathrm{nM}\right)$ but temporary inhibitor of trypsin (10). Trypsin inhibitory activity has also been found for natural domain 5 and recombinant domains 8 and 15 (17). In contrast, no proteinase has been found to be inhibited by domain 1 so far, a fact that can be explained on a structural level (19). Despite their significant sequence homology and conserved disulfide pattern, LEKTI domains 1 and 6 exhibit different folding topologies and domain 1 is lacking the canonical loop structure typical of proteinase inhibitors. The three-dimensional structure of domain 6 consists of two helices and a $\beta$-hairpin structure, closely resembling the classical Kazal-type serine proteinase inhibitor fold, including the inhibitory binding loop. In domain 1 , the putative $\mathrm{P} 1^{\prime}$ site is followed by a short 310 -helix that bends into a long $\mathrm{COOH}$-terminal $\alpha$-helix, thus lacking the $\beta$-hairpin observed in domain 6 (19).

In this study, we constructed the chimeric LEKTI domain Dom1PI to investigate the determinants of the structure and activity differences. Most LEKTI domains and other serine proteinase inhibitors contain a conserved sequence motif consisting of a proline followed by a $\beta$-branched aliphatic amino acid in the $\mathrm{P}^{\prime}$ and $\mathrm{P}^{\prime}$ position. This motif is found in 13 of 15 LEKTI domains, including domain 6, but is absent from LEKTI domain 1. By substituting Pro and Ile into the corresponding positions of LEKTI domain 1 (Phe28Pro/Phe29Ile), we produced a chimeric protein (Dom1PI). The three-dimensional structure of Dom1PI reveals the existence of a chameleon sequence of seven residues and indicates the importance of nonlocal interactions to the secondary structure of domain 1 .

\section{EXPERIMENTAL PROCEDURES}

Expression and Purification of Dom1PI. The procedure used for the expression and purification of Dom1PI is similar to that previously described for domain 1 of LEKTI (20). In brief, we used the vector construct pET-32a-Xa-Dom1 and performed a QuikChange mutagenesis (Stratagene, La Jolla, CA) to generate the vector pET-32a-Xa-Dom1PI. This construct produces Dom1PI with an $\mathrm{NH}_{2}$-terminal Escherichia coli thioredoxin fusion (Trx tag, Novagen, Madison, WI) connected by a 43-amino acid residue linker containing six histidine residues and a factor Xa cleavage site. To ensure accurate disulfide formation, an E. coli tr $x \mathrm{~B}^{-} /$gor $522^{-}$double mutant [E. coli Origami (DE3), Novagen] with an oxidative cytoplasm was used $(21,22)$. This strategy has been shown to result in the correct LEKTI domain 1 disulfide pattern (20). The soluble fraction of the cell lysate was used for further purification, using one-step $\mathrm{Co}^{2+}$-chelating affinity chromatography (23). The Trx tag and the linker region were removed by factor Xa cleavage. This strategy resulted in a homogeneous product with correct disulfide bonds as proven by RP-HPLC, mass spectrometry, and NMR. For uniform ${ }^{15} \mathrm{~N}$ labeling, Dom1PI was isolated and purified from $E$. coli Origami (DE3) cultures grown in M9 minimal medium (24) enriched with ${ }^{15} \mathrm{NH}_{4} \mathrm{Cl}$, using the same purification protocol described above. As the expression strain lacks the capability of synthesizing leucine, ${ }^{15} \mathrm{~N}$-labeled L-leucine (Campo Scientific, Veenendaal, The Netherlands) was added to the medium according to the manufacturer's instructions (Novagen).

Proteinase Inhibition and Cleavage Assays. The inhibitory activity of Dom1PI was assessed by determining its effects on the enzymatic activity of the serine proteinases bovine trypsin and chymotrypsin, human $\beta$-tryptase, thrombin, coagulation factor Xa, plasmin, urokinase-type plasminogen activator, kallikrein 5 and leukocyte elastase, porcine tissue kallikrein and pancreatic elastase, and Bacillus subtilis subtilisin. Briefly, the proteinases were incubated in the absence or presence of $10 \mu \mathrm{M}$ Dom1PI (inhibitor/enzyme ratio $>250$ for all proteinases) at room temperature in assay buffer [50 mM Tris, $150 \mathrm{mM} \mathrm{NaCl}, 0.01 \%$ Triton X-100, $0.01 \%$ sodium azide $(\mathrm{pH} 7.6$ ), and $0.1 \mu \mathrm{g} / \mathrm{mL}$ heparin for stabilization of $\beta$-tryptase]. The residual enzymatic activity was quantified both immediately after addition of Dom1PI and after preincubation for 30 min by following the hydrolysis of a suitable 7-amino-4-methylcoumarin substrate for 10 min in a Perkin-Elmer HTS 7000 microtiter plate fluorometer.

To determine whether trypsin or chymotrypsin cleaves Dom1PI, a $10 \mu \mathrm{M}$ Dom1PI solution was incubated for 30 min at room temperature with buffer alone or the proteinases $(0.6 \mathrm{nM}$, i.e., the concentration used in the inhibition kinetic experiments, or $1 \mu \mathrm{M}$ ). Subsequently, the $\mathrm{NH}_{2}$-terminal amino acid sequences were determined using an Applied Biosystems Procise 492 sequencer.

NMR Spectroscopy and Data Processing. All NMR experiments were carried out at $298 \mathrm{~K}$ on a commercial Bruker DRX600 spectrometer equipped with a tripleresonance ${ }^{1} \mathrm{H},{ }^{13} \mathrm{C},{ }^{15} \mathrm{~N}$ probe and pulsed field gradient capabilities. For homonuclear two-dimensional NMR spectra, the sample contained approximately $1.5 \mathrm{mM}$ unlabeled protein in $0.5 \mathrm{~mL}$ of NMR buffer $[10 \mathrm{mM}$ potassium phosphate $(\mathrm{pH} 5.0)$ and $10 \%(\mathrm{v} / \mathrm{v}) \mathrm{D}_{2} \mathrm{O}$ ] or buffered $\mathrm{D}_{2} \mathrm{O}$ $(99.994 \%)$. The sample for heteronuclear three-dimensional NMR spectra contained approximately $1.3 \mathrm{mM}{ }^{15} \mathrm{~N}$-labeled protein in $0.3 \mathrm{~mL}$ of NMR buffer. Homonuclear DQF-COSY, TOCSY ( $80 \mathrm{~ms}$ mixing time), and NOESY (150 and 200 ms mixing times) and heteronuclear ${ }^{15} \mathrm{~N}$ HSQC, HNHA, ${ }^{15} \mathrm{~N}$ TOCSY-HSQC (60 ms mixing time), ${ }^{15} \mathrm{~N}$ NOESY-HSQC (150 ms mixing time) (25), and ${ }^{15} \mathrm{~N}$ HMQC-NOESY-HSQC (150 ms mixing time) spectra were recorded using standard techniques (26, and references cited therein). $\left\{{ }^{1} \mathrm{H}\right\}^{15} \mathrm{~N}$ NOE values were measured using the pulse sequence of Dayie and Wagner (27) with a relaxation delay of $6 \mathrm{~s}$; the evolution time increments in the indirect dimension of the spectra with and without proton saturation by application of a train of $120^{\circ}$ high-power pulses for the final $3 \mathrm{~s}$ of the relaxation delay were recorded alternately in a single combined experiment. 
Homonuclear spectra data were multiplied with a squared sine-bell window function shifted by $\pi / 4$ prior to Fourier transformation. Sixth-order baseline and phase corrections were used. Data processing was performed using the NDee software package (SpinUp Inc., Dortmund, Germany) operating on Sun workstations. Heteronuclear data were processed using software written in-house and visualized using NMRView version 5.0.4 (28) and the NDee software package (SpinUp Inc.) on Sun workstations. Data processing consisted of linear prediction, apodization with $\pi / 2$ - or $\pi / 3$ shifted squared sine-bell functions, zero filling in all dimensions, and Fourier transformation. Baseline correction in the acquisition dimension was performed using a model free algorithm (29). The proton chemical shifts were referenced to external DSS at $0.0 \mathrm{ppm}$. The chemical shifts of the ${ }^{15} \mathrm{~N}$ resonances were referenced indirectly using an $\omega_{\mathrm{N}} / \omega_{\mathrm{H}}$ ratio of 0.101329118 at $298 \mathrm{~K}(30)$.

Sequence-specific resonance assignment was carried out using characteristic NOE cross-peaks from ${ }^{15} \mathrm{~N}$ NOESYHSQC and ${ }^{15} \mathrm{~N}$ HMQC-NOESY-HSQC spectra. The ${ }^{15} \mathrm{~N}$ TOCSY-HSQC spectrum was used to assign intraresidual proton resonances. ${ }^{1} \mathrm{H} \alpha$ chemical shifts were taken from HNHA and ${ }^{15} \mathrm{~N}$ TOCSY-HSQC spectra.

The distance restraints for structure calculation were taken from the ${ }^{15} \mathrm{~N}$ NOESY-HSQC spectrum for NOEs involving amide protons and from the homonuclear NOESY spectra for NOEs involving aliphatic and aromatic protons. ${ }^{3} J\left(\mathrm{H}^{\mathrm{N}}, \mathrm{H}^{\alpha}\right)$ coupling constants were obtained from line-shape analysis of the antiphase cross-signal splitting in a high-resolution DQF-COSY spectrum using a Lorentzian function for peak fitting as well as from cross-peak to diagonal peak intensity ratios in the HNHA spectrum.

Structure Calculation. In total, 368 nontrivial interresidual unambiguous NOESY cross-peaks were used for structure calculation (Table 1). According to their relative intensities, these cross-peaks were divided into three groups and converted into upper distance constraints: strong (2.7 $\AA$ ), medium $(3.5 \AA)$, and weak $(5.0 \AA)$. The Karplus equation was used to convert ${ }^{3} J\left(\mathrm{H}^{\mathrm{N}}, \mathrm{H}^{\alpha}\right)$ coupling constants into $\phi$ angles allowing the following deviations. ${ }^{3} J\left(\mathrm{H}^{\mathrm{N}}, \mathrm{H}^{\alpha}\right)$ coupling constants of $<6.0 \mathrm{~Hz}$ were translated into $\phi$ angle constraints of $-60 \pm 20^{\circ}$, and coupling constraints of $>8.0 \mathrm{~Hz}$ were translated into $\phi$ angle constraints of $-120 \pm 40^{\circ}$. Disulfide bonds were taken into account by using one NOE distance restraint $\left(d_{\mathrm{ss}}=2.02 \pm 0.10 \AA\right)$ for each disulfide bond.

All structures were calculated using X-PLOR version 3.851 (31) and a modified ab initio simulated annealing protocol. The structure calculation strategy is similar to those described previously, i.e., a three-stage simulated annealing protocol (32), floating assignments of prochiral groups (33), and a modified conformational database potential term (34, 35 ). The conformational search phase ( 80 ps of molecular dynamics at $2000 \mathrm{~K}$ ) was followed by a refinement in which the system was cooled to $1000 \mathrm{~K}$ within $60 \mathrm{ps}$. During this period, the force constants for nonbonded interactions were increased to their final value. In a second cooling phase, the system was cooled from 1000 to $100 \mathrm{~K}$ within $30 \mathrm{ps}$. To approach the energy minimum, 1200 steps of energy minimization were performed, the final 1000 steps being without conformational database potential.

Initial ambiguities in the NOE cross-peak assignments were resolved by an iterative approach using several rounds

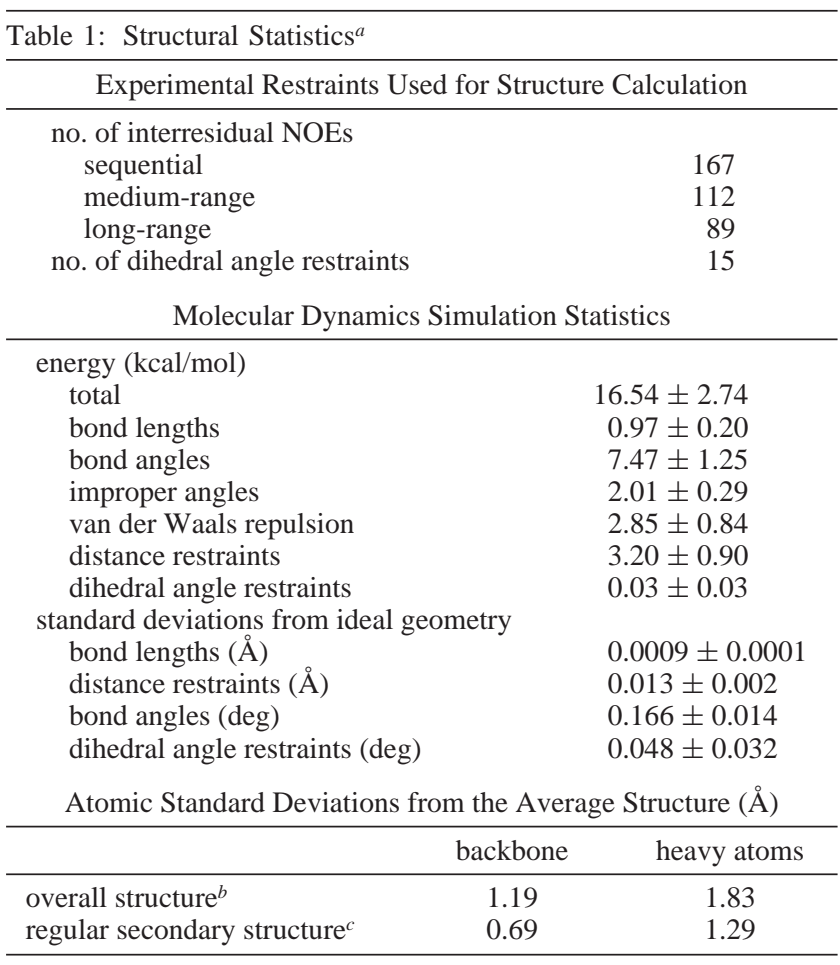

${ }^{a}$ Except for the experimental restraints, all values are average values from the 30 accepted structures in the form average value \pm standard deviation. ${ }^{b}$ Residues $1-55 .{ }^{c}$ Residues $3-13,15,16,19,20,30,31$, 35,36 , and $39-52$.

of structure calculations with subsequent distance analysis. Each round of structure calculation started from templates with randomized backbone torsion angles. Dihedral angle constraints were introduced in later rounds of the structure calculation. Control calculation without disulfide bond distance restraints and without the conformational database potential term resulted in virtually the same overall structure.

A family of 160 structures was calculated, and 30 structures were selected according to the lowest overall energy for further characterization. Geometries of all structures, as well as elements of secondary structure, were analyzed using MOLMOL (36) and PROCHECK (37). MOLMOL (36) was used for visualization of the structure.

Data Bank Entries. The coordinates and NMR restraints have been deposited in the Protein Data Bank (PDB entry 1uuc), and chemical shift values and coupling constants have been deposited in the BioMagResBank (BMRB entry 6110).

\section{RESULTS AND DISCUSSION}

Structure Determination. A complete sequence-specific assignment of the backbone as well as side chain resonances of Dom1PI was obtained from homonuclear and heteronuclear spectra. The existence of unambiguous $\mathrm{H} \alpha(i)$ Pro$\mathrm{H} \delta(i+1)$ nuclear Overhauser effect (NOE) signals in the NOE spectroscopy (NOESY) spectra and the fact that $\mathrm{H} \alpha$ (i) - Pro-H $\alpha(i+1)$ connectivities were absent (38) identified a trans conformation for the prolyl peptide bonds of Pro23 and Pro28, respectively.

A total number of 368 distance constraints, comprising 167 sequential, 112 medium-range, and 89 long-range NOE contacts, as well as 15 dihedral angle constraints (Table 1) were used for structure calculations. Both ${ }^{15} \mathrm{~N}$ NOESYHSQC and homonuclear spectra were used to obtain these 


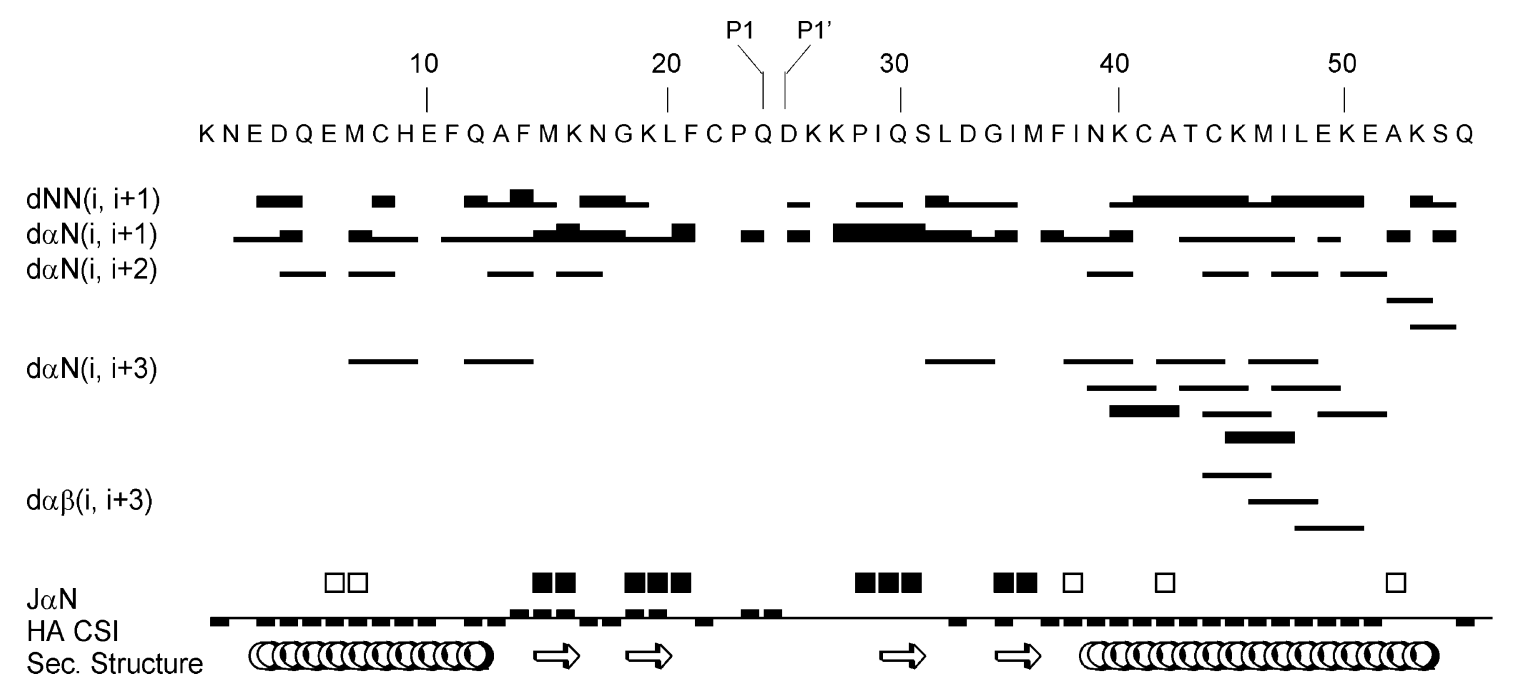

FIGURE 1: Secondary structure of Dom1PI. Summary of sequential and medium-range NOEs, coupling constants, and chemical shifts. The relative strength of the NOEs, classified as weak, medium, and strong, from cross-peak intensities of the ${ }^{15} \mathrm{~N}$ NOESY-HSQC spectrum and two-dimensional NOESY spectra, is indicated by the height of the horizontal bars. ${ }^{3} J\left(\mathrm{H}^{\mathrm{N}}, \mathrm{H}^{\alpha}\right)$ values of $<6$ and $>8 \mathrm{~Hz}$, typical of helical and extended structure, are shown as white and black squares, respectively. Positive and negative chemical shift indices that are typical of extended and helical structure are denoted with rectangles above and below the axis. Elements of regular secondary structure of Dom1PI, as derived from structure calculations, are shown at the bottom. The $\mathrm{P} 1-\mathrm{P} 1^{\prime}$ site is indicated in the sequence.

distance constraints. A total of $26{ }^{3} J\left(\mathrm{H}^{\mathrm{N}}, \mathrm{H}^{\alpha}\right)$ coupling constants were determined by using a high-resolution doublequantum-filtered correlated spectroscopy (COSY) spectrum and the HNHA spectrum. Of these, 15 coupling constants could be unambiguously transformed into $\phi$ angle constraints with five helix-typical coupling constants $\left(\phi=-60 \pm 20^{\circ}\right)$ being located within the two helices. Another 10 coupling constants characteristic of extended structure $(\phi$ angle $=$ $\left.-120 \pm 40^{\circ}\right)$ were found around Met15-Phe21 and Ile29Met36 (Figure 1). These data are consistent with characteristic medium-range NOEs that suggest the existence of two $\beta$-hairpin motifs. In the final round of structure calculation, a set of 160 structures was calculated. The 30 structures showing the lowest overall energies were used to represent the solution conformation of Dom1PI (Figure 2a). A control calculation without disulfide bond distance restraints confirmed the disulfide pattern. The PROCHECK analysis (37) shows a favorable backbone conformation for almost all residues of the 30 structures with $89.4 \%$ of the non-glycine and non-proline residues in the most favored regions and $9.1 \%$ in additional allowed regions of the Ramachandran plot.

The best-fit superposition of the structures (Figure 2a), as well as the corresponding local rmsd values (Figure 3a), demonstrates a well-defined tertiary structure for Dom1PI, except for the loop region from Gln24 to Lys27. The structures have no distance violations greater than $0.2 \AA$, and there are no systematic violations greater than $0.12 \AA$.

Description of the Tertiary Structure of Dom1PI. Dom1PI consists of two $\alpha$-helices with an almost orthogonal orientation, two $\beta$-hairpin structures, and a hydrophobic core that position the putative proteinase binding loop comprising residues Cys22-Ile29 (Figure 2b). The $\mathrm{NH}_{2}$-terminal helix extends from Glu3 to Ala13 and is followed by a $\beta$-hairpin from Met15 to Leu20. A second $\beta$-hairpin motif extends from Gln30 to Met36 and merges into a long $\mathrm{COOH}$-terminal $\alpha$-helix from Asn39 to Lys53 that displays characteristic $d_{\alpha N^{-}}$ $(i, i+4)$ NOEs. The potential $\mathrm{P} 1-\mathrm{P} 1^{\prime}$ site (Gln24 and Asp25) is located in an exposed loop connecting both $\beta$-hairpins (Figures 1 and 2). As calculated from the best-fit superposi- tion of 30 structures, the rmsd value for the complete sequence is $1.19 \AA$ for the backbone heavy atoms and 1.83 $\AA$ for all heavy atoms. Regions with regular secondary structure exhibit rmsd values of $0.69 \AA$ for the backbone heavy atoms and $1.29 \AA$ for all heavy atoms (Table 1). The rmsd values per residue (Figure $3 a$ ) indicate a higher level of local disorder for the exposed potential binding loop than for the regular secondary structure. Also, the values of the $\left\{{ }^{1} \mathrm{H}\right\}{ }^{15} \mathrm{~N}$ heteronuclear NOEs for the amide protons of Cys22-Ile35 are less than 0.6, indicating an increased inherent flexibility of the $\mathrm{N}-\mathrm{H}$ vectors on a pico- to nanosecond time scale (39; Figure 3c). This is in accordance with a high uncertainty in the $\psi$ and $\phi$ angles of P3-P3' residues (Cys22-Lys27, respectively; Table 2). A hydrophobic core is built by the disulfide bonds and Phe11, Phe14, Leu20, and Leu48. These residues display a high number of long-range NOE connectivities among each other (Figure 3b).

The Secondary and Tertiary Structure of DomlPI Is Different from That of the Wild-Type Protein and Resembles Domain 6. (1) Comparison of Dom1PI with Wild-Type LEKTI Domain 1. The sequence of Dom1PI differs from that of the wild-type protein, LEKTI domain 1, in only two sequentially adjacent amino acids, so both proteins are $96 \%$ identical in sequence. However, the order and arrangement of elements of secondary structure, as well as the overall tertiary structure of Dom1PI, reveal remarkable differences from the wild-type protein. Dom1PI lacks the short 310 -helix present in domain 1 (19), instead displaying a loop conformation in this region. Furthermore, Dom1PI exhibits a second $\beta$-hairpin structure (Figures $2 \mathrm{~b}$ and $4 \mathrm{a}$ ). The amino acids participating in this $\beta$-hairpin are distant from the position of mutation and are part of the long $\mathrm{COOH}$-terminal $\alpha$-helix in the wild-type protein (Figure 4a). Similarities between Dom1PI and the wild-type protein exist in the first $\beta$-hairpin motif comprising residues Met15-Leu20, in parts of the $\mathrm{NH}_{2}$-terminal and $\mathrm{COOH}$-terminal $\alpha$-helices, and in the hydrophobic core that is formed by both disulfide bonds, Phe11, Phe14, Leu20, and Leu48. Compared to the case with wild-type domain 1, the unambiguous assignment of all 55 

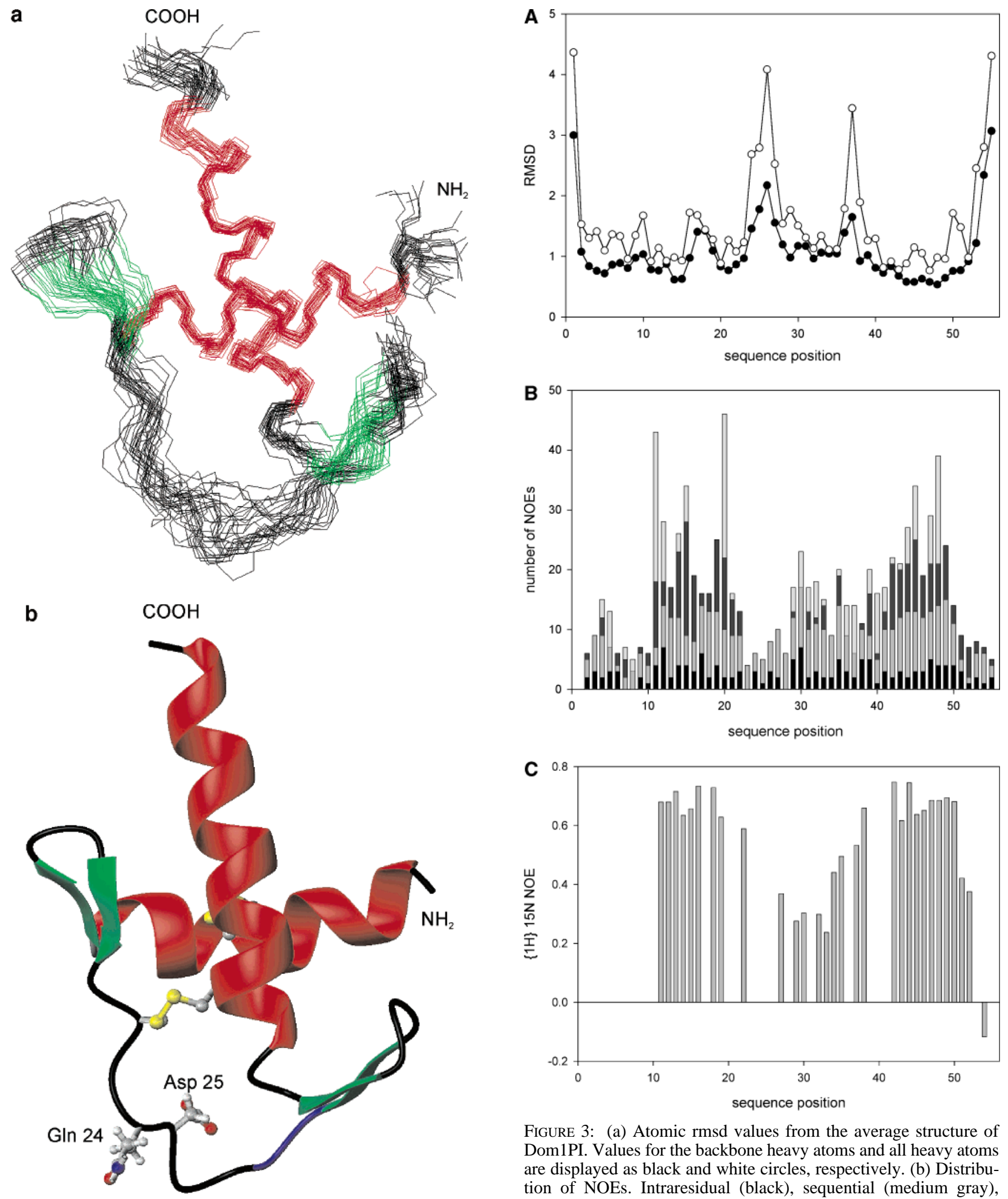

FIGURE 3: (a) Atomic rmsd values from the average structure of Dom1PI. Values for the backbone heavy atoms and all heavy atoms are displayed as black and white circles, respectively. (b) Distribution of NOEs. Intraresidual (black), sequential (medium gray), medium-range (dark gray), and long-range (light gray) NOEs are displayed as vertical stacked bars. (c) $\left\{{ }^{1} \mathrm{H}\right\}{ }^{15} \mathrm{~N}$ NOE values at 600 $\mathrm{MHz}$ are displayed as vertical bars.

FIGURE 2: (a) Best-fit superposition of the 30 lowest-energy structures of Dom1PI [generated with MOLMOL (36)]. Helical elements are colored red, and $\beta$-sheet structure is colored green. (b) Schematic drawing of a representative structure of Dom1PI indicating elements of regular secondary structure. Helical elements are colored red; $\beta$-sheet structure is colored green, and the mutation site is colored blue. The heavy side chain atoms of the putative $\mathrm{P} 1-\mathrm{P} 1^{\prime}$ site (Gln24 and Asp25, respectively) and both disulfide bonds $(\mathrm{C} 8-\mathrm{C} 44$ and $\mathrm{C} 22-\mathrm{C} 41)$ are shown.

spin systems leads to a better structural definition of the termini of Dom1PI [overall backbone rmsd $=1.19 \AA$ (Dom1PI) vs $1.80 \AA$ (wild-type domain 1)] and causes an extension of the $\mathrm{NH}_{2}$-terminal $\alpha$-helix.

The changes in the secondary structure of the central part of the protein induced by the mutation become apparent in 
Table 2: $\phi$ and $\psi$ Angles of P3 to P3' Residues of LEKTI Domain 1, Domain 6 (19), and Dom1PI in Comparison to Characteristic Backbone Angle Values of the Canonical Binding Loop of Proteinase Inhibitors (46)

\begin{tabular}{|c|c|c|c|c|c|}
\hline position & angle & canonical loop & Dom1PI & domain 1 & domain 6 \\
\hline \multirow[t]{3}{*}{ P3 } & & & Cys & Cys & Cys \\
\hline & $\phi$ & -140 to $-120^{\circ}$ & $-92.1 \pm 23.7^{\circ}$ & $-51.9 \pm 3.8^{\circ}$ & $-136.9 \pm 26.7^{\circ}$ \\
\hline & $\psi$ & 140 to $170^{\circ}$ & $93.8 \pm 24.6^{\circ}$ & $-46.5 \pm 2.5^{\circ}$ & $179.2 \pm 14.9^{\circ}$ \\
\hline \multirow[t]{3}{*}{$\mathrm{P} 2$} & & & Pro & Pro & Thr \\
\hline & $\phi$ & -100 to $-60^{\circ}$ & $-61.2 \pm 3.1^{\circ}$ & $-62.8 \pm 1.6^{\circ}$ & $-105.6 \pm 19.8^{\circ}$ \\
\hline & $\psi$ & 140 to $180^{\circ}$ & $173.7 \pm 60.8^{\circ}$ & $79.9 \pm 4.5^{\circ}$ & $-176.5 \pm 8.4^{\circ}$ \\
\hline \multirow[t]{3}{*}{$\mathrm{P} 1$} & & & Gln & Gln & Arg \\
\hline & $\phi$ & -120 to $-95^{\circ}$ & $-77.4 \pm 40.7^{\circ}$ & $-118.6 \pm 8.7^{\circ}$ & $-84.5 \pm 16.3^{\circ}$ \\
\hline & $\psi$ & 10 to $50^{\circ}$ & $-92.2 \pm 48.3^{\circ}$ & $-79.5 \pm 40.8^{\circ}$ & $21.2 \pm 38.9^{\circ}$ \\
\hline \multirow[t]{3}{*}{$\mathrm{P} 1^{\prime}$} & & & Asp & Asp & Glu \\
\hline & $\phi$ & -100 to $-60^{\circ}$ & $-78.0 \pm 58.2^{\circ}$ & $-135.0 \pm 43.7^{\circ}$ & $-52.9 \pm 61.7^{\circ}$ \\
\hline & $\psi$ & 140 to $180^{\circ}$ & $130.4 \pm 39.7^{\circ}$ & $68.9 \pm 7.0^{\circ}$ & $150.3 \pm 13.9^{\circ}$ \\
\hline \multirow[t]{3}{*}{$\mathrm{P} 2^{\prime}$} & & & Lys & Lys & Asn \\
\hline & $\phi$ & -140 to $-100^{\circ}$ & $-74.6 \pm 23.1^{\circ}$ & $-61.8 \pm 5.9^{\circ}$ & $-110.7 \pm 21.5^{\circ}$ \\
\hline & $\psi$ & 70 to $120^{\circ}$ & $-24.8 \pm 76.6^{\circ}$ & $-8.7 \pm 5.1^{\circ}$ & $59.2 \pm 6.6^{\circ}$ \\
\hline \multirow[t]{3}{*}{$\mathrm{P} 3^{\prime}$} & & & Lys & Lys & Asp \\
\hline & $\phi$ & -140 to $-100^{\circ}$ & $-95.8 \pm 42.6^{\circ}$ & $-41.7 \pm 0.9^{\circ}$ & $-142.1 \pm 60.6^{\circ}$ \\
\hline & $\psi$ & 70 to $120^{\circ}$ & $120.8 \pm 27.6^{\circ}$ & $-26.9 \pm 0.7^{\circ}$ & $78.8 \pm 7.9^{\circ}$ \\
\hline
\end{tabular}

comparing the $\mathrm{H} \alpha$ secondary chemical shifts (40) of both proteins (Figure 5). The resonances of residues 25-28 and 35-39 are shifted to lower field in Dom1PI compared to those in wild-type domain 1, whereas for residues 24,31 , and 34, a shift to higher field can be observed. This suggests a change in secondary structure in the corresponding positions. Shifts to lower field in the region preceding the mutation site indicate an extended conformation and the decomposition of the short 310 -helix. In the center of this $3_{10}$-helix, Phe28 was replaced with proline, leading to a backbone $\phi$ angle usually not found in helices. Another reason for the disappearance of the $3_{10}$-helix might be a loss of stabilization of an intrinsically unstable helix by loss of hydrophobic contacts. The $\mathrm{NH}_{2}$-terminal end of the 310 -helix in wild-type domain 1 comprises two lysines (19) that amplify the helix dipole, therefore being energetically unfavorable (41). The 310 -helix, however, is stabilized by contacts between Phe 29 and Ile38 that are part of the long COOH-terminal $\alpha$-helix in the wild-type protein. Via replacement of Phe 28 and Phe29 with Pro and Ile, respectively, the introduction of Pro changes the orientation of Ile so that stabilization by hydrophobic contact is omitted and the $310^{-}$ helix can no longer be built.

Shifts to lower field in the region distant from the mutation site (residues 35-39) indicate an extended conformation corresponding to the second $\beta$-hairpin in the overall structure (Figures 4a and 5). Thus, the mutation causes a change not only in the secondary structure in the proximal region but also in a distant region, indicating an influence on tertiary contacts.

(2) Comparison of DomlPI with LEKTI Domain 6. Although the level of sequence identity between Dom1PI and LEKTI domain 6 is only 31\%, their folding topology is very similar (Figure $4 b$ ). The length and orientation of both terminal $\alpha$-helices, as well as of the first $\beta$-hairpin structure, are almost identical $(\mathrm{rmsd}=1.54 \AA)$. Only the orientation of the second $\beta$-hairpin toward the helices is different (Figure 4b). Another similarity in Dom1PI and LEKTI domain 6 is the existence of an exposed potential canonical loop (Figures $2 \mathrm{a}, \mathrm{b}$ and $4 \mathrm{~b}$ ). In domain 6 , this region acts as a proteinase binding loop (42), leading to temporary trypsin inhibitory activity that is most likely due to enhanced flexibility (19).
As for domain 6 of LEKTI (19), no medium- and long-range distance restraints could be observed for residues Gln24Lys27 within the putative canonical loop region of Dom1PI (Figure 3b), also leading to a less well-defined backbone geometry compared to the rest of the protein (Figure 3a,c). We suggest that this level of local disorder (Figure 3a) and inherent flexibility (Figure 3c) are even higher than those of domain 6 , leading to an only marginal inhibitory activity of Dom1PI (see below).

Proteinase Inhibition and Cleavage. Despite the structural similarity to LEKTI domain 6, an efficient but temporary trypsin inhibitor, Dom1PI, has no effect on the enzymatic activity of trypsin even at a concentration of $10 \mu \mathrm{M}$, i.e., an inhibitor/enzyme ratio of $>15000$. The lack of inhibition cannot be attributed to a temporary or slow inhibition mechanism because inhibition was not observed after different incubation times $(0-30 \mathrm{~min})$ and cleavage of the protein could not be detected under the conditions used for inhibition kinetics. After incubation with higher trypsin concentrations (1 $\mu \mathrm{M}, 30 \mathrm{~min})$, however, cleavage at the Lys19-Leu20 bond and to a lesser degree at the Lys40Cys41 bond was observed, indicating that these regions are accessible to the proteinase.

Dom1PI also does not inhibit plasmin and subtilisin, proteinases inhibited by recombinant LEKTI (43) and a recombinant LEKTI fragment containing domains 6-8 and a part of domain 9, which shows only weak inhibition of plasmin (44). Also, $\beta$-tryptase, the coagulation factors thrombin and $\mathrm{Xa}$, urokinase-type plasminogen activator, the kallikreins pK1 (porcine tissue kallikrein) and hK5 (human stratum corneum tryptic enzyme), and pancreatic elastase are not inhibited. Marginal effects on the activity of chymotrypsin and leukocyte elastase, however, were observed $(\sim 10 \%$ inhibition at $10 \mu \mathrm{M})$, indicating that Dom1PI shows very weak inhibitory activity against these proteinases. Fragmentation of Dom1PI could be detected only at higher chymotrypsin concentrations $(1 \mu \mathrm{M})$ at the Phe21-Cys22 and Phe37-Ile38 bonds. In comparison, recombinant LEKTI inhibits chymotrypsin to a similar degree, whereas the affinity for leukocyte elastase is much higher $\left(K_{\mathrm{i}} \sim 300 \mathrm{nM}\right)(43)$. It is worth mentioning that, in contrast to isolated LEKTI domains, full-length recombinant LEKTI shows noncompeti- 
a
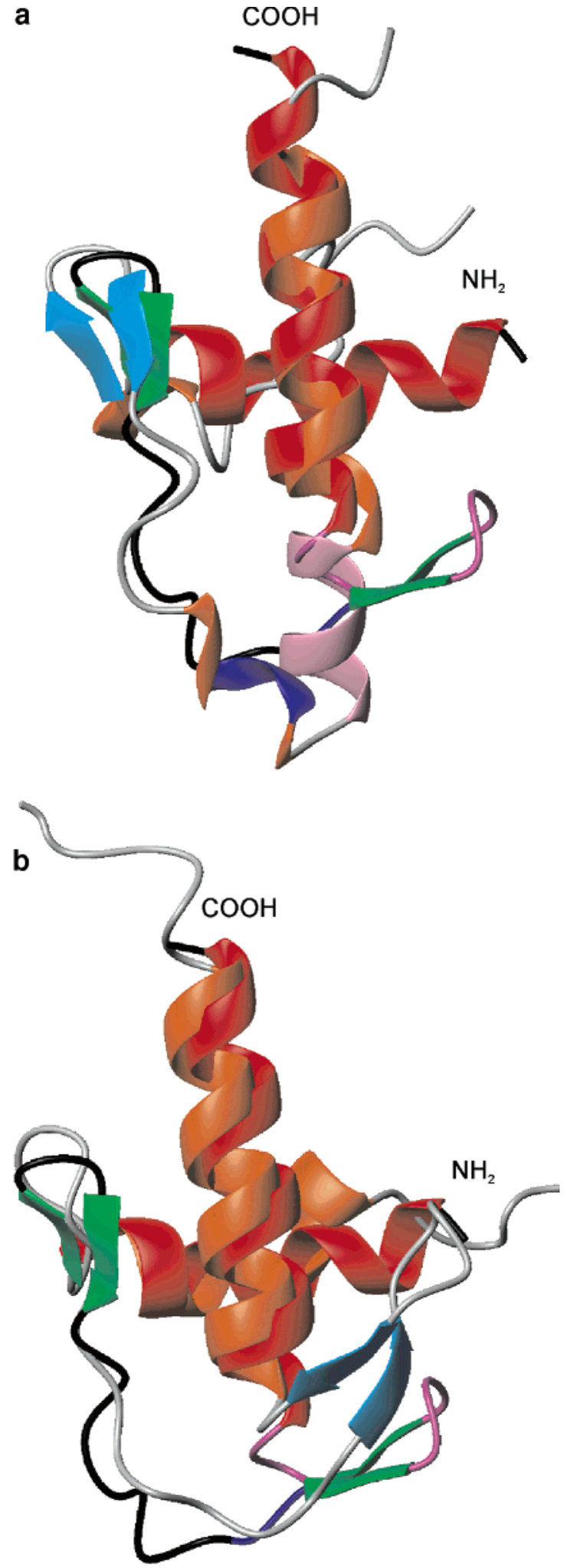

FIGURE 4: (a) Schematic drawing of the best-fit superposition of Dom1PI and wild-type domain 1. For Dom1PI, the same color code as in Figure $2 \mathrm{~b}$ was used. For wild-type domain 1, the helical elements are colored orange and the $\beta$-sheets cyan. The mutation site is colored blue. The chameleon sequence is colored violet. The $\beta$-sheet in Dom1PI (green) is an integral part of the chameleon sequence. (b) Schematic drawing of the best-fit superposition of Dom1PI and domain 6. For Dom1PI, the same color code as in Figure $4 \mathrm{a}$ was used. For domain 6, the helical elements are colored orange and $\beta$-sheets cyan. In Dom1PI, the mutation site is colored blue and the chameleon sequence violet. The $\beta$-sheet in Dom1PI (green) is an integral part of the chameleon sequence.

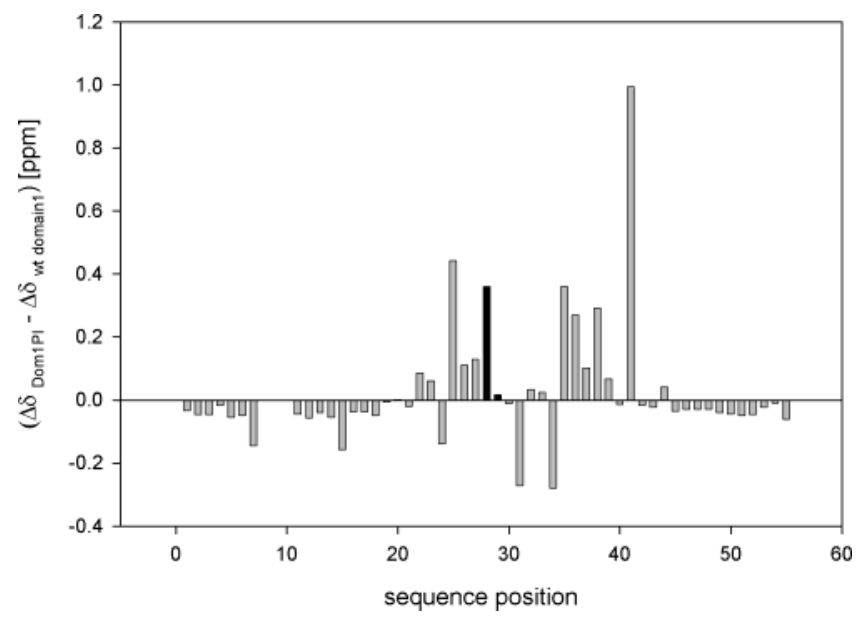

FIGURE 5: $\mathrm{H} \alpha$ chemical shift differences between domain 1 and Dom1PI. To account for different random coil shifts of the mutated amino acids (black), secondary chemical shifts were used for the calculation of chemical shift differences of Dom1PI and wild-type domain 1 . For residues $8-10$, no values have been acquired since they have not been assigned in wild-type domain 1 .

tive inhibition of various proteinases, suggesting the existence of a binding site distinct from the catalytic center of the proteinase (43).

The observed lack of strong inhibitory activity of Dom1PI might be due to intrinsic flexibility, unfavorable residues or unfavorable backbone angles (Table 2 ) in the $\mathrm{P} 3-\mathrm{P} 3^{\prime}$ region, the orientation of the second $\beta$-hairpin motif (Figure $4 b$ ), or a combination thereof. Canonical serine proteinase inhibitors are characterized by a canonical binding loop that interacts with a complementary strand of the proteinase forming a stabilized antiparallel $\beta$-sheet (45). To perform this task, the backbone torsion angles along the binding loop must adopt characteristic values (46; Table 2). A scaffold comprising secondary structure elements, a hydrophobic core, and disulfide bonds stabilizes the position of the binding loop of proteinase inhibitors (47). In contrast to domain 6, some of the backbone angles of the binding loop residues of Dom1PI differ from those found for the typical canonical loop conformation. Due to ambiguous coupling constants and the absence of NOEs, these angles are poorly defined (Table 2 and panels a and b of Figure 3). This result is in accordance with a higher intrinsic flexibility in this region as determined by $\left\{{ }^{1} \mathrm{H}\right\}{ }^{15} \mathrm{~N}$ heteronuclear NOEs (Figure $3 \mathrm{c}$ ).

The second $\beta$-hairpin motif in Dom1PI and domain 6 positions the putative binding loop by providing a stabilizing scaffold together with the first $\beta$-hairpin motif, a hydrophobic core, and both disulfide bridges. The best-fit superposition of Dom1PI and domain 6, however, indicates that the second $\beta$-hairpin motif connecting the putative binding loop with the $\mathrm{COOH}$-terminal $\alpha$-helix is oriented in different ways (Figures $4 \mathrm{~b}$ and 6). We suggest that this difference originates from the sequence in the kink region between the hairpin and the $\mathrm{COOH}$-terminal helix: residue 38 is an isoleucine in Dom1PI, whereas domain 6, several other LEKTI domains, and many other Kazal-type serine proteinase inhibitors exhibit a glycine at the corresponding position. Glycine enables the protein backbone to form bends or turns by allowing a large range of torsion angles. In the case of Dom1PI, the voluminous side chain of Ile38 prevents the formation of a sharp kink such as that found for domain 6 (Figure 6). This side chain could be a reason for the absence 


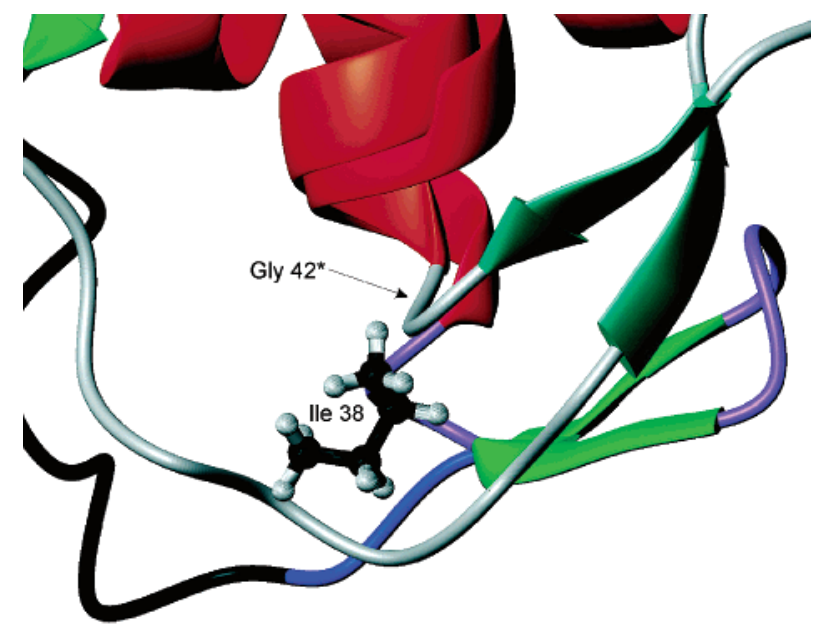

FIGURE 6: Close-up of the best-fit superposition of Dom1PI and domain 6 (same color code as in Figure $4 \mathrm{~b}$ ). The transition region between the $\beta$-hairpin motif and the $\mathrm{COOH}$-terminal $\alpha$-helix is shown. For Dom1PI, the side chain atoms of Ile38 are shown and the corresponding position of Gly42* in domain 6 is marked with an arrow.

of inhibitory activity of Dom1PI, acting by preventing the required orientation of the second $\beta$-hairpin and thus indirectly of the putative binding loop.

Also, the unusual P1 residue of Dom1PI cannot be excluded as another reason for the lack of strong inhibitory activity. In general, Kazal-type proteinase inhibitors which act against trypsin seem to prefer positively charged amino acids in this position (48), which is consistent with a substrate-like mechanism of inhibition since trypsin cleaves after Arg and Lys. In Dom1PI, this positive charge is missing as the P1 site is occupied by glutamine. With this P1 residue Dom1PI may be active against a different target proteinase, as it exhibits some characteristics typical of canonical serine proteinase inhibitors. On the other hand, main chain-main chain contacts and therefore the backbone geometry are much more important to proteinase-inhibitor interactions than side chain interactions and therefore the actual P1 residue (49). For example, mutational studies with proteinase-inhibitor complexes revealed that despite replacement of the P1 residue with other amino acids the same residue position served as the P1 site independent of the particular amino acid (50-52).

On the basis of the marginal inhibitory activity against chymotrypsin and leukocyte elastase and of the geometrical features of the potential canonical loop of Dom1PI, we predict that Dom1PI exhibits some characteristics of a proteinase inhibitor but that further mutations are needed to turn it into a potent inhibitor of these proteinases. At present, however, the possibility that Dom1PI might exhibit high inhibitory activity against a proteinase not tested in this study cannot be ruled out.

Identification of a Chameleon Sequence. A chameleon sequence is characterized by a context-dependent secondary structure that is stabilized by nonlocal interactions (4). By a comparison of the overall structures of Dom1PI and wildtype domain 1 (Figure 4a), the existence of a chameleon sequence becomes manifest. The sequence stretch from Leu32 to Ile38 displays an $\alpha$-helical conformation in wildtype domain 1 but a $\beta$-hairpin motif in Dom1PI. This sequence is not affected by the mutation and is identical in both proteins. The direction of the $\alpha \rightarrow \beta$ transition is worth mentioning as most presently known chameleon sequences have been discovered by converse transitions $(5,53) . \alpha \rightarrow$ $\beta$ transitions are particularly interesting with regard to prion diseases $(8)$.

The 310 -helix in domain 1 seems to be stabilized by interactions between Phe29 and Ile38. Therefore, we suspect that this hydrophobic interaction stabilizes two intrinsically unstable helices. This hypothesis is confirmed by the helix propensity prediction algorithm AGADIR (54), resulting in helical propensities of $<2 \%$ for both the $3_{10}$-helix of domain 1 and the chameleon sequence (Leu32-Ile38). This implies that the $\mathrm{NH}_{2}$-terminal extension of the $\mathrm{COOH}$-terminal $\alpha$-helix of domain 1 built by the chameleon sequence is intrinsically unstable and stabilized by tertiary contacts. Such a stabilization of secondary structure elements by tertiary contacts is presumed to be a general principle in protein folding (55). We assume that after substitution of one interaction partner the stabilization energy decreases so that Dom1PI adopts the energetically preferred $\beta$-hairpin structure. A small difference in the energies of different conformations that can be offset by tertiary interactions is also postulated for several other chameleon sequences $(56,57)$.

The identification of mutations causing a structural change could be very relevant for the understanding and prediction of amyloid diseases (58) as several mutations involved in prion diseases are situated in elements of regular secondary structure $(59,60)$. The structure of Dom1PI determined in this study demonstrates that minor mutations can cause extensive nonlocal structural changes.

\section{ACKNOWLEDGMENT}

We thank N. Herz, U. Persau, R. Hofmann, and K. Schweimer for expert technical assistance and helpful discussions. We are grateful to D. Gabrijelcic-Geiger, S. Streicher, and R. Mentele for performing inhibition kinetics and amino acid sequencing.

\section{REFERENCES}

1. Haber, E., and Anfinsen, C. B. (1961) Regeneration of enzyme activity by air oxidation of reduced subtilisin-modified ribonuclease, J. Biol. Chem. 236, 422-424.

2. Anfinsen, C. B. (1973) Principles that govern the folding of protein chains, Science 181, 223-230.

3. Mezei, M. (1998) Chameleon sequences in the PBD, Protein Eng. $11,411-414$.

4. Minor, D. L., Jr., and Kim, P. S. (1996) Context-dependent secondary structure formation of a designed protein sequence, Nature 380, 730-734.

5. Yang, W., Ko, T., Corselli, L., Johnson, R. C., and Yuan, H. S. (1998) Conversion of a $\beta$-strand to an $\alpha$-helix induced by a singlesite mutation observed in the crystal structure of Fis mutant Pro ${ }^{26}$ Ala, Protein Sci. 7, 1875-1883.

6. Abel, K., Yoder, M. D., Hilgenfeld, R., and Jurnak, F. (1996) An $\alpha$ to $\beta$ conformational switch in EF-Tu, Structure 4, 1153-1159.

7. Carr, C. M., and Kim, P. S. (1993) A spring-loaded mechanism for the conformational change of influenza hemagglutinin, Cell $73,823-832$.

8. Harrison, P. M., Bamborough, P., Daggett, V., Prusiner, S. B., and Cohen, F. E. (1997) The prion folding problem, Curr. Opin. Struct. Biol. 7, 53-59.

9. Prusiner, S. B. (1994) Molecular biology and genetics of prion disease, Philos. Trans. R. Soc. London, Ser. B 343, 447-463.

10. Mägert, H. J., Ständker, L., Kreutzmann, P., Zucht, H. D., Reinecke, M., Sommerhoff, C. P., Fritz, H., and Forssmann, W. G. (1999) LEKTI, a novel 15-domain type of serine proteinase inhibitor, J. Biol. Chem. 274, 21499-21502. 
11. Chavanas, S., Garner, C., Bodemer, C., Ali, M., Hamel-Teillac, D., Wilkinson, J., Bonafe, J. L., Paradisi, M., Kelsell, D. P., Ansai, S., Mitsuhashi, Y., Larregue, M., Leigh, I. M., Harper, J. I., Taieb, A., de Prost, Y., Cardon, L. R., and Hovnanian, A. (2000) Localization of the Netherton syndrome gene to chromosome 5q32, by linkage analysis and homozygosity mapping, Am. J. Hum. Genet. 66, 914-921.

12. Walley, A. J., Chavanas, S., Moffatt, M. F., Esnouf, R. M., Ubhi, B., Lawrence, R., Wong, K., Abecassis, G. R., Jones, E. Y., Harper, J. I., Hovnanian, A., and Cookson, W. O. (2001) Gene polymorphism in Netherton and common atopic disease, Nat. Genet. 29, 175-178.

13. Sprecher, E., Chavanas, S., DiGiovanna, J. J., Amin, S., Nielsen, K., Prendiville, J. S., Silverman, R., Esterly, N. B., Spraker, M. K., Guelig, E., de Luna, M. L., Williams, M. L., Buehler, B., Siegfried, E. C., Van Maldergem, L., Pfendner, E., Bale, S. J., Uitto, J., Hovnanian, A., and Richard, G. J. (2001) The spectrum of pathogenic mutations in SPINK5 in 19 families with Netherton syndrome: implications for mutation detection and first case of prenatal diagnosis, Invest. Dermatol. 117, 179-187.

14. Komatsu, N., Takata, M., Otsuki, N., Ohka, P., Amano, O., Takehara, K., and Saijoh, K. (2002) Elevated stratum corneum hydrolytic activity in Netherton syndrome suggests an inhibitory regulation of desquamation by SPINK5-derived peptides, J. Invest. Dermatol. 118, 436-443.

15. Bitoun, E., Bodemer, C., Amiel, J., de Prost, Y., Stoll, C., Calvas, P., and Hovnanian, A. (2002) Prenatal diagnosis of a lethal form of Netherton syndrome by SPINK5 mutation analysis, Prenatal Diagn. 22, 121-126.

16. Mägert, H. J., Kreuzmann, P., Ständker, L., Walden, M., Drögemüller, K., and Forssmann, W. G. (2002) LEKTI: a multidomain serine proteinase inhibitor with pathophysiological relevance, Int. J. Biochem. Cell Biol. 34, 573-576.

17. Mägert, H. J., Kreuzmann, P., Drögemüller, K., Ständker, L., Adermann, K., Walden, M., John, H., Korting, H. C., and Forssmann, W. G. (2002) The 15-domain serine proteinase inhibitor LEKTI: biochemical properties, genomic organization, and pathophysical role, Eur. J. Med. Res. 7, 49-56.

18. Ahmed, A., Kandola, P., Ziada, G., and Parenteau, N. (2001) Coronavirus main proteinase $\left(3 \mathrm{CL}^{\mathrm{pro}}\right)$ structure: basis for design of anti-SARS drugs, J. Protein Chem. 20, 273-278.

19. Lauber, T., Schulz, A., Schweimer, K., Adermann, K., and Marx, U. C. (2003) Homologous proteins with different folds: the threedimensional structures of domains 1 and 6 of the multiple Kazaltype inhibitor LEKTI, J. Mol. Biol. 328, 205-219.

20. Lauber, T., Marx, U. C., Schulz, A., Kreutzmann, P., and Rösch, P. (2001) Accurate disulfide formation in Escherichia coli: overexpression and characterization of the first domain (HF6478) of the multiple Kazal-type inhibitor LEKTI, Protein Expression Purif. 22, 108-112.

21. Derman, A. I., Prinz, W. A., Belin, D., and Beckwith, J. (1993) Mutations that allow disulfide bond formation in the cytoplasm of Escherichia coli, Science 262, 1744-1747.

22. Prinz, W. A., Aslund, F., Holmgren, A., and Beckwith, J. (1997) The role of the thioredoxin and glutaredoxin pathways in reducing protein disulfide bonds in the Escherichia coli cytoplasm, J. Biol. Chem. 272, 15661-15667.

23. Porath, J. (1992) Immobilized metal ion affinity chromatography, Protein Expression Purif. 3, 263-281.

24. Sambrook, J., Fritsch, E. F., and Maniatis, M. (1989) Molecular cloning: a laboratory manual, Cold Spring Harbor Laboratory Press, Plainview, NY.

25. Talluri, S., and Wagner, G. (1996) An Optimized 3D NOESYHSQC, J. Magn. Reson., Ser. B 112, 200-205.

26. Cavanagh, J., Fairbrother, W. J., Palmer, A. G., III, and Skelton, N. J. (1996) Protein NMR Spectroscopy, Academic Press, San Diego.

27. Dayie, K. T., and Wagner, G. (1994) Relaxation-rate measurements for ${ }^{15} \mathrm{~N}-{ }^{1} \mathrm{H}$ groups with pulsed field gradients and preservation of coherence pathways, J. Magn. Reson., Ser. A 111, 121-126.

28. Johnson, B. A., and Blevins, R. A. (1994) NMRView: A computer program for the visualization and analysis of NMR data, J. Biomol. NMR 4, 603-614.

29. Friedrichs, M. (1995) A model-free algorithm for the removal of baseline artefacts, J. Biomol. NMR 5, 147-153.

30. Markley, J. L., Bax, A., Arata, Y., Hilbers, C. W., Kaptein, R., Sykes, B. D., Wright, P. E., and Wüthrich, K. (1998) Recommendations for the presentation of NMR structures of proteins and nucleic acids, Pure Appl. Chem. 70, 117-142.
31. Brünger, A. T. (1993) X-PLOR version 3.1: A system for X-ray crystallography and NMR, Howard Hughes Medical Institute and Yale University Press, New Haven, CT.

32. Nilges, M. (1993) A calculation strategy for the structure determination of symmetric dimers by ${ }^{1} \mathrm{H}$ NMR, Proteins: Struct., Funct., Genet. 17, 297-309.

33. Holak, T. A., Nilges, M., and Oschkinat, H. (1989) Improved strategies for the determination of protein structures from NMR data: the solution structure of acyl carrier protein, FEBS Lett. $242,649-654$

34. Neudecker, P., Sticht, H., and Rösch, P. (2001) Improving the efficiency of the Gaussian conformational database potential for the refinement of protein and nucleic acid structures, J. Biomol. NMR 21, 373-375.

35. Kuszewski, J., and Clore, G. M. (2000) Sources of and solutions to problems in the refinement of protein NMR structures against torsion angle potentials of mean force, J. Magn. Reson. 146, 249254.

36. Koradi, R., Billeter, M., and Wüthrich, K. (1996) MOLMOL: a program for display and analysis of macromolecular structures, J. Mol. Graphics 14, 51-55.

37. Laskowski, R. A., MacArthur, M. W., Moss, D. S., and Wright, P. E. (1993) PROCHECK: a program to check the stereochemical quality of protein structures, J. Appl. Crystallogr. 26, 283-291.

38. Wüthrich, K. (1986) NMR of Proteins and Nucleic Acids, John Wiley \& Sons, New York.

39. Kay, L. E., Torchia, D. A., and Bax, A. (1989) Backbone dynamics of proteins as studied by ${ }^{15} \mathrm{~N}$ inverse detected heteronuclear NMR spectroscopy: application to staphylococcal nuclease, Biochemistry 28, 8972-8979.

40. Wishart, D. S., Sykes, B. D., and Richards, F. M. (1991) Relationship between nuclear magnetic resonance chemical shift and protein secondary structure, J. Mol. Biol. 222, 311-333.

41. Shoemaker, K. R., Kim, P. S., York, E. J., Stewart, J. M., and Baldwin, R. L. (1987) Tests of the helix dipole model for stabilization of $\alpha$-helices, Nature 326, 563-567.

42. Kreutzmann, P., Schulz, A., Ständker, L., Forssmann, W.-G., and Mägert, H. J. (2004) Recombinant production, purification and biochemical characterization of domain 6 of LEKTI: a temporary Kazal-type-related serine proteinase inhibitor. J. Chromatogr., B: Anal. Technol. Biomed. Life Sci. 803, 75-81.

43. Mitsudo, K., Jayakumar, A., Henderson, Y., Frederick, M. J., Kang, Y., Wang, M., El-Naggar, A. K., and Clayman, G. L. (2003) Inhibition of serine proteinases plasmin, trypsin, subtilisin A, cathepsin G, and elastase by LEKTI: a kinetic analysis. Biochemistry 42, 3874-3881.

44. Jayakumar, A., Kang, Y., Mitsudo, K., Henderson, Y., Frederick, M. J., Wang, M., El-Naggar, A. K., Marx, U. C., Briggs, K., and Clayman, G. L. (2004) Expression of LEKTI domains 6-9' in the baculovirus expression system: recombinant LEKTI domains 6-9' inhibit trypsin and subtilisin A. Protein Expression Purif. $35,93-101$.

45. Bode, W., Wei, A.-Z., Huber, R., Meyer, E., Travis, J., and Neumann, S. (1986) X-ray crystal structure of the complex of human leukocyte elastase (PMN elastase) and the third domain of the turkey ovomucoid inhibitor, EMBO J. 5, 2453-2458.

46. Bode, W., and Huber, R. (1992) Natural protein proteinase inhibitors and their interaction with proteinases, Eur. J. Biochem. 204, 433-451.

47. Bode, W., and Huber, R. (2000) Structural basis of the endoproteinase-protein inhibitor interaction, Biochim. Biophys. Acta 1477 , $241-252$

48. Laskowski, M., Jr. (1998) http://www.chem.purdue.edu/ LASKOWSKI/\#data.

49. Jackson, R. M. (1999) Comparison of protein-protein interactions in serine protease-inhibitor and antibody-antigen complexes: implications for the protein docking problem, Protein Sci. 8, 603613.

50. Lu, W., Apostol, I., Qasim, M. A., Warne, N., Wynn, R., and Zhang, W. L. (1997) Binding of amino acids side-chains to S1 cavities of serine proteases, J. Mol. Biol. 266, 441-461.

51. Fujinaga, M., Huang, K., Bateman, K. S., and James, M. N. (1998) Computational analysis of the binding of $\mathrm{P} 1$ variants of domain 3 of turkey ovomucoid inhibitor to Streptomyces griseus protease B, J. Mol. Biol. 284, 1683-1694.

52. Helland, R., Otlewski, J., Sundheim, O., Dadlez, M., and Smalas, A. O. (1999) The crystal structures of the complexes between bovine $\beta$-trypsin and ten P1 variants of BPTI, J. Mol. Biol. 287, 923-942. 
53. Dalal, S., Balasubramanian, S., and Regan, L. (1997) Protein alchemy: Changing $\beta$-sheet into $\alpha$-helix, Nat. Struct. Biol. 4, 548551.

54. Munoz, V., and Serrano, L. (1994) Elucidating the folding problem of $\alpha$-helical peptides using empirical parameters, II. Helix macrodipole effects and rational modification of the helical content of natural peptides, J. Mol. Biol. 245, 275-296.

55. Daggett, V., and Fersht, A. R. (2003) Is there a unifying mechanism for protein folding? Trends Biochem. Sci. 28, 18-25.

56. Sagermann, M., Gay, L., and Metthews, B. W. (2003) Longdistance conformational changes in a protein engineered by modulated sequence duplication, Proc. Natl. Acad. Sci. U.S.A. 100, 9191-9195.

57. Ikeda, K., Galzitskaya, O. V., Nakamura, H., and Higo, J. (2003) $\beta$-Hairpins, $\alpha$-helices, and the intermediates among the secondary structures in the energy landscape of a peptide from a distal $\beta$-hairpin of SH3 domain, J. Comput. Chem. 24, 310-318.

58. Dobson, C. M. (2001) The structural basis of protein folding and its links with human disease, Philos. Trans. R. Soc. London, Ser. B 356, 133-145.

59. Riek, R., Hornemann, S., Wider, G., Billeter, M., Glockshuber, R., and Wüthrich, K. (1996) NMR structure of the mouse prion protein domain PrP (121-231), Nature 382, 180-182.

60. Young, K., Piccardo, P., Dloughy, S., Bugiani, O., Tagliavini, F., and Ghetti, B. (1999) The Human Genetic Prion Diseases, in Prions: Molecular and Cellular Biology (Harris, D. A., Ed.) pp 139-175, Horizon Scientific Press, Wymondham, U.K.

BI0492399 